\title{
Parasite annexins - new molecules with potential for drug and vaccine development
}

Andreas Hofmann, ${ }^{1 *}$ Asiah Osman, ${ }^{1}$ Chiuan Yee Leow, ${ }^{2,3,4}$ Patrick Driguez, ${ }^{2}$ Donald P. McManus ${ }^{2}$ and Malcolm K Jones ${ }^{2,3}$

${ }^{1}$ Structural Chemistry Program, Eskitis Institute for Cell \& Molecular Therapies, Griffith University, Nathan, Queensland, 4111, Australia

${ }^{2}$ Queensland Institute of Medical Research, Herston Queensland, 4029, Australia

${ }^{3}$ School of Veterinary Sciences, The University of Queensland, Queensland, 4072, Australia

${ }^{4}$ Institute for Research in Molecular Medicine, Universiti Sains Malaysia, 11800 Penang, Malaysia

*Correspondence to: Andreas Hofmann, Structural Chemistry, Eskitis Institute for Cell \& Molecular Therapies, Griffith University, N75 Don Young Road, Brisbane Innovation Park, Nathan, Brisbane, Qld 4111, Australia

Email: a.hofmann@griffith.edu.au; Telephone/Facsimile: +61-7-3735-4425

Manuscript: BioEssays, 2nd revised version, 02/04/11, p_19.doc

Word count: 8854 
In recent years, annexins have been discovered in several nematodes and other parasites, and distinct differences between the parasite annexins and those of the hosts make them potentially attractive targets for anti-parasite therapeutics.

Annexins are ubiquitous proteins found in almost all organisms across all kingdoms. Here, we present an overview of novel annexins from parasitic organisms, and summarise their phylogenetic and biochemical properties, with a view to using them as drug or vaccine targets. Building on structural and biological information that has been accumulated for mammalian and plant annexins, we describe a predicted additional secondary structure element found in many parasite annexins that may confer unique functional properties, and present a unique antigenic epitope for use as a vaccine.

Keywords: Annexins; drug discovery; helminths; infectious diseases; nematodes; parasites; vaccine design

Abbreviations: $\quad$ ANX, annexin; ATP, adenosine triphosphate; EST, expressed sequence tag; TSP2, Tetraspanin 2; VSP, Variant-specific Surface Protein.

\section{Introduction}

Parasites of humans and animals belong to a diverse range of eukaryotic clades spanning two Kingdoms, the Metazoa and Protista. The pathways to parasitism have seemingly been adopted in many lineages of organisms in different events. As different parasite groups can be traced back in their evolutionary history to free-living progenitors, it is not surprising that each major group of parasites displays unique, but perhaps convergent, adaptations to the strictures of life within or on a host. Many remarkable morphological and molecular adaptations have accompanied the adoption of parasitism, as witnessed by the extraordinary attachment armature displayed by trypanorhynch cestodes, the sophisticated extracellular membrane complexes in erythrocyte-inhabiting malaria or 
the bizarre egg hatching behaviour of the blood fluke Schistosoma japonicum.[1-3] The richly diverse morphological, behavioural and developmental alterations in parasites are matched by the evolution of distinctive and characteristic protein families. Most often, however, the molecules expressed by parasitic groups have high sequence identity with those expressed by their free-living relatives, or their hosts. How these ubiquitous molecules are used in parasitism is a question of major interest. One family of proteins, abundant and diverse in parasites and hosts alike, is the annexin family.

Annexins are widespread throughout the Eukaryota. This protein family has become apparent in molecular studies of diverse parasite groups, ranging from the diplomonad protist Giardia to the neodermatan platyhelminths Taenia and Schistosoma. Although their prominence, especially in schistosomes, may prove to be an artifact of investigation, the annexins seem uncommonly dissimilar in some parasite groups. Since their identification, parasite annexins have been linked to potential biotechnological or therapeutic applications, most notably as vaccine and drug targets.

Mechanistically, a passive and an active role of annexins in parasites has been observed to help the survival prospects of the invading pathogens. In organisms such as Giardia and schistosomes, the annexins appear to be integral for the stability of the parasite and thus help protect against structural breakdown in hostile environment and upon immune attack by the host. For the plant nematode Heterodera and the platyhelminth Taenia, secreted annexins have been reported that actively downregulate the immune response by the host.[4,5] At the same time, annexins in parasites are frequently found in areas prone to exposure to the host's immune system and thus fulfill the criteria of potential vaccine targets.

This review serves to outline new information on annexins from parasitic organisms and the implications for novel curative applications. 


\section{Annexins}

Some 12 annexins are described for vertebrates, and have been assigned the identifiers A1 to A13 (Annexin A12 is unassigned). Annexins from other taxa are assigned identifiers that reflect major phylogenetic lineages. Thus, family B annexins are found in invertebrates (and are hence termed B1-13 based on evolutionary ancestry with vertebrate annexins); family $\mathrm{C}$ are found in fungi, stramenopiles and alveolates; family D are from true plants; family E from other protists, notably the diplomonads;[6] and family F from bacteria.

Annexins are soluble proteins with the hallmark feature of calcium-dependent binding to phospholipid membranes. All known members of this protein family possess a distinctive, highly conserved fold, the annexin fold.[7] This fold enables general adapter-like functions at the surface of phospholipid membranes.[8] The presence of this fold gives annexins diverse roles including vesicular transport (endo- and exocytosis), and membrane attachment of the cytoskeleton (reviewed in [9]). Several annexins have also been implicated in extracellular functions,[10] but always with involvement of membrane surfaces.

The ability of annexins to bind phospholipids and proteins has implicated these molecules in a range of functional activities, including the formation of rafts, ceramide binding, biosensing of membrane topography, cell signaling events including calcium regulation, regulation of Ras, detection of membrane stress and membrane repair.[11] Not all mammalian annexins have all of these functions, and not all are expressed in all cell types.

The canonical calcium-dependent membrane binding known from mammalian annexins is believed to be based purely on formation of a ternary complex between protein, calcium and membrane, facilitated by the endonexin sequence present in each repeat on the convex side of the molecule (see Figure 1). From studies with plant annexins, it became clear that the general membrane binding mechanism differs in various annexin sub-families of the annexins, since the lack of the endonexin 
sequence in some repeats is compensated for by basic and hydrophobic residues on the convex, membrane-binding side which interact with the membrane directly.[12-14] Our preliminary studies on some parasite annexins indicate a similar situation prevails.[15]

Some mammalian annexins as well as alpha giardins have been reported to possess lectin properties.[16,17] This ability to interact with oligosaccharides is of particular interest, for it is hypothesised that externalised parasite annexins may use glycosaminoglycan binding as a means of attachment to host tissue. Our ongoing studies with alpha giardins, and schistosome and tapeworm annexins indicate that this is not a commonly shared property among parasite annexins ([15] and Osman et al., unpublished).

\section{Annexins of Parasites}

\section{Protista}

The protists are unicellular eukaryotes and represent the stem group for evolution of all multicellular eukaryotes. Annexin evolution generally follows that of protists and multicellular clades.[6] A annexins are found in most protist clades, but are apparently absent from euglenozoans (kinetoplastids and euglenids) and radiolarians. Annotation search of the genomic datasets of the human parasitic kinetoplastids Trypanosoma and Leishmania, did not reveal any annexin domains for these organisms. In addition, annexins have not been found for malaria, but are present in other alveolatans including the parasitic ciliate Entodinium,[18] and Perkinsus marinus, a parasite of oysters thought now to be a dinoflagellate.[19] Annexins appear to be expressed in all other protistan clades, including cercozoans, particularly those most closely related to plants, fungi and metaozoans, but are poorly known. Annexins are present in fungi, including the yeast Yarrowia lipolytica.[20] 


\section{Annexins of Giardia}

Most studies of annexins from protists have focused on the alpha giardins, peculiar proteins assigned to the family E annexins expressed by the diplomonad Giardia. The Giardia genome dataset (http://giardiadb.org) lists 21 different alpha giardins, (numbered alpha-1 -19, with alpha-7 existing in three variants (alpha-7.1, 7.2, 7.3). Giardia is an intestinal parasite that occurs worldwide and in all climatic zones.[21,22] This water-borne pathogen is a major cause of human diarrheal diseases, which collectively have been ranked second only to respiratory infections in terms of mortality worldwide.[23] The Giardia life cycle involves a parasitic and trophic stage, the trophozoite, and an inert transmissive cystic form.[24] The cytoskeleton of Giardia has been recognised as an important factor of virulence,[25] since cell motility, the ability to attach to the mucosa, and the resistance against degradation by bile salts are important aspects of its survival. The central role of alpha giardins is thought to be a stabilising one, preventing membrane breakdown and structural collapse of the parasite in its extremely hostile environment.[26] On emergence from the cyst in the host intestine, the parasite must first assemble its ventral adhesive disk (an organelle used in attachment to the intestinal mucous) and the flagella from fragments stored in the cyst.[25] These dramatic structural changes occurring during cytokinesis in encystation and excystation could potentially allow for certain alpha giardins being externalised and may also explain the high immunogenicity of some alpha giardins observed during acute giardiasis.[27]

\section{Annexins in the Metazoa}

Annexins are widespread in the Metazoa, occurring in all groups examined so far. The molecules occur in modern relatives of the hypothesised stem group, the choanoflagellates,[28] and multiple members occur in basal metazoans of the phylum Placozoa (Trichoplax adhaerens), the Porifera (Ephydatia fluviatilis) and Cnidaria (Nematostella vectensis).[29,30] In E. fluviatilis, ANX(Ef) is enriched in choanocytes, the primary nutritive cells that line the spongocoel (internal gut-like cavity), and which have cellular morphology highly reminiscent of choanoflagellates.[29] The high 
expression of this annexin in choanocytes and archeocytes enables early differentiation of the primitive lining cells to be traced. Phylogenetic analysis of annexin amino acid sequences (Figure 2) from a range of lower invertebrates including $T$. adherens indicated an early bifurcation of placozoan annexins, but there was insufficient evidence to suggest the placozoan proteins are paralogous.

\section{Annexins in nematodes: the free-living Caenorhabditis elegans and parasitic species}

Four annexins, named nex-1 -4 , have been identified in the free-living nematode C. elegans.[31] While nex-1 and nex-3 are expressed continuously in the developmental stages of $C$. elegans, the expression of nex-2 and nex-4 was reported to be only temporal.[32] The highest expressed member of this protein family, nex-1, is present in many structures in the worm.[33] Ultrastructural studies of nex-1 showed it to be localised to the spermathecal valve, a structure that represents the final gate for the egg on its passage from the ovary to the spermathecal chamber. Since the cell must stretch open to enable passage of the egg, the surrounding membranes are folded up and need to unfold to provide the required surface area for the expansion. Nex-1 has therefore been hypothesised to mediate the reversible membrane contractions required for the valve action,[34] very much reflecting what was already proposed for mammalian annexin-membrane interactions (e.g. endoand exocytosis). While nex-1, -2 and -3 have been shown to possess lipid binding and lectin properties, nex-4 displays neither such capacity.[32]

Annexins of animal-parasitic nematodes have been poorly investigated, and only two annexins of plant pathogenic nematodes have been described. An annexin from the potato cyst nematode Globodera pallida has been found in the reproductive organs,[35] reminiscent of nex-1 localisation in C. elegans. The soybean cyst nematodes Heterodera glycines and H. schachtii secrete an annexin through their secretory cells of the oesophageal gland.[4] Cumulative evidence indicates that these secreted annexins act to down-regulate plant responses to invading pathogens, thereby enhancing 
the survival prospects of the invading worms.

Recent genomic sequencing of the filarial nematode Brugia malayi, an agent of human filariasis, has uncovered a range of annexins.[36] Phylogenetic analyses of some invertebrate annexin sequences suggest that of the Brugia annexins, $\operatorname{ANX}(\mathrm{Bm}) 2$ bifurcates as a probable orthologue with

nex-4 of C. elegans (Figure 2). Nex-4 is expressed early in development, with highest levels found in first stage larvae.[32] Although the functional relevance of nex-4 is undetermined, there is the possibility of the existence of annexins specific to the microfilarial stage of filarial nematodes, functional characterisation of which may shed light on the biology of microfilariae in their human hosts and their early development in mosquito vectors.

\section{Platyhelminthes - Cestoda}

Cysticercosis, a human disease caused by infection with C. cellulosae, the metacestode (larval or cystic stage) of the pig tapeworm Taenia solium, is of major significance as a human disease and for the economic losses it may cause in developing countries. Although humans serve as the definitive host, harbouring tapeworm adults in the intestine, they can also act as intermediate host, and carry the metacestode in various tissue sites, notably the central nervous system, muscles, subcutaneous tissue and the eye.[37] Human patients suffer from highly debilitating effects such as blindness, epileptic seizures, and brain lesions.[38]

Annexin B1 was identified in the cyst stage of T. solium,[39] and its expression seems to be tissuedependent.[40] Its existence in cystic fluid, as well as in host tissue surrounding the region of infiltration and in the host serum suggested the protein is secreted. Taenia annexin B1 was reported to induce calcium-dependent apoptosis of eosinophils.[5] Notably, annexin A1 of mammals is known to act as one signal presented by apoptotic cells to induce their removal by phagocytes.[41] Taenia annexin B1 may thus act to mimic a common host pathway to reduce the inflammatory response to cysticercosis. 
Two more annexins, B2 and B3 have been identified in T. solium, and based on available ESTs, further annexins probably exist in this species. All Taenia annexin sequences bifurcate with schistosome annexins, 1,3, and 5/6 (Figure 2), which are predominant in the epithelia of the tegument (body lining) and other epithelia of these blood flukes.[42,43]

\section{Platyhelminthes - Digenea}

Schistosomes are the most important of the human helminths in terms of morbidity and mortality, and a recent meta-analysis by King assigned 2-15\% disability weight to this human disease..[44] Recent estimates of the global disease burden for schistosomiasis indicate that some 207 million people are infected, with over 750 million people at risk.[45]

Annexins are particular noteworthy as surface associated molecules of adult schistosomes. These blood flukes are protected in their human host by a highly ordered syncytial cytoplasm, the tegument, that performs multiple functions of protection, immune evasion, nutrition, and signaling.[46] The surface membrane of the tegument is covered by an additional trilaminate membrane layer, the so-called membranocalyx. The structure and composition of the membranocalyx remains unclear at present, but among its functions is an unusual property of sequestering glycolipids from host erythrocytes.[47] It is currently proposed that the membranocalyx provides a physical barrier that protects the parasite from immune attack.

In a proteomic study using surface biotinylation, a paucity of proteins was revealed in the membrane-membranocalyx complex of the schistosome tegument.[42] Among the few proteins present are tetraspanin 2 (TSP2), annexin, and a secreted protein of unknown function, Sm29. $\operatorname{ANX}(\mathrm{Sm}) 3$ and its orthologue from $S$. japonicum, $\mathrm{ANX}(\mathrm{Sj}) 3$ were among the most abundant proteins found in surface membrane extracts.[43] Two other less abundant annexins found at the surface of S. japonicum were ANX(Sj)1 and ANX(Sj)5.[43] Annexins are likely to be an abundant surface-related molecule of digeneans, as the human liver fluke, Opistorchis viverrini, also 
expresses abundant surface annexins.[48]

Draft genomes for $S$. mansoni and S. japonicum have recently been reported and a first pass annotation is available (http://www.schistodb.org).[49,50] In a recent survey of the databases, we identified 14 annexins from S. mansoni, 6 from S. japonicum and 1 from S. haematobium.

Of these, it is known that schistosome annexins 1,3 and 5 are expressed in the tegument of the adult parasites.[42,43] A tissue-specific transcriptomic survey of female $S$. japonicum indicated that different annexins were expressed preferentially by different cell types, with the gut lining expressing annexins 1 and 5, while the vitelline gland expressed annexin 4.[51] The abundance of annexins 1 and 5 in the gut and tegument suggests that these molecules may be epithelial annexins in these parasites.

\section{Platyhelminthes - Monogenea}

Monogeneans are skin and gill parasites of fishes, with a simple one-host life cycle. The haematophagic polyopistocotylean Microcotyle sebastis, a parasite of the rockfish Sebastes schlegeli in Korea, causes severe mortality in its hosts. Recently a single annexin, here called ANX(Ms), was identified and cloned from M. sebastis.[52] Phylogenetic analyses suggest that ANX(Ms), although clearly divergent, bifurcates with other epithelial annexins of schistosomes and Taenia solium. Localisation of ANX(Ms) to the gut lining of M. sebastis, among other locations, lends support to the identity of this annexin as a flatworm epithelial annexin that also possesses the property of calcium-dependent binding to acidic phospholipid vesicles.[52]

\section{Unique structural features of parasite annexins}

From the structure-based sequence alignment of selected invertebrate annexins (data not shown), the highly conserved annexin fold is obvious for most of these proteins. In agreement with findings from mammalian annexins, the length of the $\mathrm{N}$-terminal domains is variable and only few parasite 
annexins with medium or long N-terminal domains are predicted to possess ordered secondary structures in these regions, including annexins $(\mathrm{Sm}) 6,(\mathrm{Sm}) 7,(\mathrm{Sm}) 8,(\mathrm{Sj}) 1$ and $(\mathrm{Sj}) 7$, as well as alpha-7, alpha-10 and alpha-16 giardin. In the annexin fold, the N-terminal domain is located at the concave, membrane-distal side of the protein, and so is the projection of the C-terminal end (Figure 1). Any structural elements fused to the $\mathrm{N}$ - or C-terminal end of the annexin fold may thus facilitate interactions with other parasite or host proteins, and may even present potential targets for therapeutic intervention. C-terminal extensions of the annexin fold with predicted additional $\alpha$ helical elements are found with alpha-8, 14 and 19 giardin, and with (Sm)11 and (Sm)12 annexin.

The endonexin sequence harbouring the canonical type II calcium binding sites are conserved in many, but not all, annexins. Schistosome annexins 7, 8 and 9, as well as the B. malayi annexins 1 and 2 do not possess any endonexin sequence. The membrane binding loop in repeat II of some parasite annexins (e.g. schistosome annexins 1-3) is likely to be enlarged due to an eight amino acid insertion between helices IIC and IID.

A unique feature of many schistosome and the three T. solium annexins is the unusually long linker region between repeats II and III, also observed with C. elegans nex-4, and alpha-12 and alpha-19 giardin (Figures 3 and 4). The typical length of such linkers in annexins is about 10-15 amino acids. In the T. solium annexins as well as in many schistosome annexins, the linker comprises between 25 and 38 amino acids. Secondary structure predictions show that the linker in annexins with 25 or more residues in that region adopts $\alpha$-helical secondary structure. We therefore anticipate that these annexins possess an additional $\alpha$-helical segment of about 10-15 amino acids on the concave side of the molecule which is generally believed to be distal to the membrane binding side. The threedimensional structure of this additional $\alpha$-helix within parasite annexins is illustrated in the comparative model of annexin B1 in Figure 1. 


\section{Annexins in Disease}

\section{Human genetic disorders}

Annexins are ubiquitous proteins, important for the health of organisms and the term “annexinopathies” has been coined for annexin-related diseases.[53] Mechanistically, the central feature of annexins in this context seems to be their ability to provide binding platforms for other proteins on membranes, and to stabilise membrane and membrane-associated structures. An example is annexin A2 which provides binding platforms for enzymes of the blood coagulation cascade.[54] A deficiency of annexin A5 in the placenta leads to recurrent pregnancy loss, and the associated conditions of anti-phospholipid antibody syndrome and Lupus anticoagulants.[55] Very recently, the membrane repair mechanisms of homo- and heterogeneous annexin assemblies have been described which occur due to high increases in calcium after disruption of the plasma membrane.[56]

\section{Annexins in viral and microbial pathogenesis}

Several viruses and parasites have infection strategies based on binding to proteoglycans in the extra-cellular matrix (e.g. influenza hemagglutinin, adhesins from Shiga toxin and Entamoeba histolytica). Repeatedly, annexins have been implicated in these processes at the interface between host and invader. A role for human annexins as viral receptors has been reported for cytomegalovirus and hepatitis B virus.[57,58]

\section{Use of annexins as anti-parasite targets}

While anti-parasite drugs offer a convenient therapy that can be effectively administered, the timely control of parasitic diseases by chemotherapy has certain drawbacks that include production costs, a requirement for repeated application following re-infection, and the induction of resistance by longterm use or lack of compliance of treatment protocols. Vaccines, on the other hand, can overcome many of these problems and offer another avenue of therapeutic intervention. 


\section{Parasite annexins as drug targets}

The primary functions of annexins known to date center around their ability to bind ultrastructural surfaces such as phospholipid membranes and their associated proteoglycan network. The timed attachment to these structures provides either mechanical protection, such as the annexin A5 protective shield in the placenta, or binding platforms, such as the annexin A2 mediated regulation of blood coagulation factors. Potential drugs therefore need to physically interfere in the binding sites of annexins to membranes, sugars and proteins, or alter the binding behaviour through allosteric mechanisms.

Oligosaccharide binding sites have been determined for annexin A5,[59] and can be mapped on parasite annexins by homology.[15] While carbohydrates usually possess low activity and insufficient drug-like properties, recent advances with glycomimetics have brought carbohydratebinding proteins into focus as novel drug targets.[60] Another approach in this context may capitalise on binding sites in the vicinity of the divalent metal ions in the membrane binding loops, since observations from plant annexins show that substrates like ATP are able to bind to the convex surface.[61,62] With appropriate compounds, it may be possible to develop drugs that can inhibit annexin interactions with membranes and/or oligosaccharides.

There is also precedence for annexins binding small molecules, namely benzo-di/thi-azepine derivatives.[63,64] These molecules bind to a pocket at the concave side of mammalian annexins (A3, A5), and have been shown to affect their interactions with phospholipid membranes in an allosteric manner. The pocket is formed on one side by the linker peptide between repeats II and III and is limited by the very $\mathrm{N}$-terminal region on the adjacent side.

Targeting parasite annexins with small molecules may offer advantages in cases where the proteins 
are not accessible or are presented at the surface. The membranocalyx of schistosomes with its paucity of proteins is an example where small molecules can provide an avenue for interference when the immune response has only a short time window of opportunity during the parasite's life cycle. Given the crucial role of alpha giardins for cytoskeletal integrity of Giardia (and probably likewise the tegumental annexins of schistosomes), drugs that disintegrate ultrastructures could prove to be highly effective therapeutics.

\section{Parasite annexins as vaccine targets}

Vaccine development for the three parasite groups considered in this review is at a different stage of advancement. For $T$. solium, encouraging recombinantly based vaccines have, for example, been developed that are highly effective in preventing infection of pigs.[65,66] The only available vaccine against giardiasis to date is a whole G. duodenalis trophozoite vaccine that showed variable results in cats and dogs,[67] and has not been successful in calves.[68] Efforts to find effective vaccines against Giardia are hampered by the fact that a particular trophozoite (the pathogenic stage of the parasite) is surrounded by one of 150 integral membrane proteins called Variant-specific Surface Proteins (VSPs) which protect against proteolysis in the small intestine.[69] Further candidates for Giardia therapeutics are the recently discovered non-variant cyst proteins and released metabolic enzymes.[70,71]

In schistosomes, the tegument (body wall) is the dominant host-parasite interactive region.[46,72] Two tegument proteins, S. mansoni tetraspanin 2 (TSP2) and Sm29, are currently considered as highly encouraging vaccine targets. Antibody recognition of TSP2 and Sm29 appears to be associated with protective immunity and resistance to schistosomiasis in human populations.[72-75] Experimental vaccination with recombinant peptides of these two molecules induced strong protection in experimental schistosomiasis, as measured by reduction in adult worm burdens and egg production,[75] but, as yet, neither has been shown to produce sterilising immunity nor 
prolonged immunological memory. Since the membranocalyx may not be a static layer but undergoes significant frequent transformation, and annexin proteins (ANX(Sm)3, ANX(Sj)3) constitute a major fraction of the proteins present in the schistosome tegument, we speculate that they could function in the control and closure of ruptures in this surface layer. This putative function for the schistosome annexins may be similar to those of their mammalian counterparts in membrane repair. In such a scenario, these proteins would also be exposed frequently to the host defence mechanisms..

The reported immunogenic properties of several parasite annexins, combined with predicted unique structural features likely exposed at the molecular level and with unique epitopes not present in mammalian annexins, may provide an exciting opportunity to pursue these antigens as vaccine targets while preventing cross-reactivity with host annexins.

\section{Conclusions and prospects}

Members of the ubiquitous protein family of annexins provide structural stabilisation, and thus are of considerable importance for the normal functioning of cellular processes. Centered around their hallmark feature of binding to phospholipid membranes, annexins fulfill these stabilising roles at membrane sites, either through mechanically reinforcing membranes, repairing membrane leaks, or providing membrane-associated platforms for enzymatic activities. Preliminary structural data available for parasite annexins to date suggest that different parasite clades possess annexins with unique properties separating them from mammalian homologues. This, coupled with the abundance of annexins in some parasites, and their likely involvement in host-parasite interactions and in host immune-modulation make them attractive targets for exploration as anti-parasite interventions.

One strategy may be to weaken or inhibit the stabilising annexin-maintained interaction networks with drugs, in order to compromise the integrity of parasites, making them vulnerable to the host 
defence mechanisms and combination treatment approaches. Alternatively, parasite annexins may also lend themselves as targets for the host immune response. Encouraging anti-helminth vaccines that have progressed to clinical trial include some that block tissue migration of the parasites and hence target molecules that are secreted into host tissues by the parasite.[76] It is uncertain whether helminth annexins comprise this class of molecules, but evidence from plant parasitic nematodes and Taenia suggest an immune modulatory role for annexins.

Some caveats need to be kept in mind, though, as we consider the anti-parasite potential of annexins. Firstly, it has been established that some annexins are among the most abundant molecules at or near the surface of parasites, such as the schistosomes. While it is speculated that these molecules play important roles in surface maintenance, such as in ensuring the integrity of the membrane-membranocalyx complex, it remains to be proven whether the molecules or domains thereof are sufficiently exposed for immune or drug mediated action.

Secondly, very little is known about the diversification, redundancy or mutability of parasite proteins and the impact these may have on parasite control strategies. To date, there have been relatively few studies assessing the effects of genetic mutations on the structure and function of parasite proteins.[77] Many metazoan parasites have astounding capacity for sexual and asexual reproduction, enabling allelic variations to be enhanced in populations, as exemplified by high densities of nucleotide polymorphisms in the genomes of outbred (naturally occurring) populations of schistosomes and Giardia.[78,79] Simple mutations have thwarted continued use of some anthelminthic compounds to control nematodes, such as Haemonchus contortus, in which a single nucleotide change renders some strains insusceptible to benzimidazoles.[80,81] By contrast, resistance in hookworms to the anthelminthic pyrantel appears to be regulated through variable transcriptional regulation of multiple family members of drug-sensitive molecules, in this case of different nicotinic acetylcholine receptor subunits.[82] Similar variable transcription of Pglycoproteins is central to insensitivity in schistosomes to the anti-schistosomal drug 
praziquantel.[83,84] Interestingly, both genetic polymorphism and transcriptional variation have been postulated to explain why a recombinant vaccine designed against a schistosome tetraspanin was ineffective in experimental schistosomiasis japonica, while efficacious in schistosomiasis mansoni. $[85,86]$

Future basic research will need to address these important issues, as well as providing further in depth studies of parasite annexins, including their tissue distribution, validation of their unique structural elements, analysis of their functional characteristics, and testing of their efficacy as vaccine and drug targets. The first steps in this direction are under way in our laboratories.

\section{Acknowledgments}

Research in AH's laboratory is supported by Griffith University, the Rebecca L Cooper Foundation, and the James N Kirby Foundation. MKJ and DPM receive funding from the Australian Research Council and National Health and Medical Research Foundation of Australia. We thank Drs Geoff Gobert and Amber Glanfield for assistance with construction of phylogenetic trees.

\section{References}

1. Olson PD, Caira JN, Jensen K, Overstreet RM, et al. 2010. Evolution of the trypanorhynch tapeworms: Parasite phylogeny supports independent lineages of sharks and rays. Int. J. Parasitol. 40: 223242.

2. Hanssen E, Carlton P, Deed S, Klonis N, et al. 2010. Whole cell imaging reveals novel modular features of the exomembrane system of the malaria parasite, Plasmodium falciparum. Int. J. Parasitol. 40: 123-134.

3. Jones MK, Bong SH, Green KM, Holmes P, et al. 2008. Correlative and dynamic imaging of the hatching biology of Schistosoma japonicum from eggs prepared by high pressure freezing. PLoS Negl. Trop. Dis. 2: e334. 
4. Patel N, Hamamouch N, Li C, Hewezi T, et al. 2010. A nematode effector protein similar to annexins in host plants. J. Exp. Bot. 40: 123-134.

5. Yan H, Xue G, Mei Q, Ding F, et al. 2008. Calcium-dependent proapoptotic effect of Taenia solium metacestodes annexin B1 on human eosinophils: A novel strategy to prevent host immune response. Int. J. Biochem. Cell Biol. 40: 2151-2163.

6. Moss SE, Morgan RO. 2004. The annexins. Genome Biol. 5: 219.

7. Hofmann A, Huber R. 2003. Structural conservation and functional versatility: allostery as a common annexin feature. In: Annexins: biological importance and annexin-related pathologies. Landes Bioscience, Georgetown, TX. pp. 38-60.

8. Benz J, Hofmann A. 1997. Annexins - From structure to function. Biol. Chem. 378: 177-183.

9. Grewal T, Enrich C. 2009. Annexins--modulators of EGF receptor signalling and trafficking. Cell Signal. 21: 847-858.

10. Siever D, Erickson H. 1997. Extracellular annexin II. Int. J. Biochem. Cell Biol. 29: 12191223.

11. Monastyrskaya K, Babiychuk E, Draeger A. 2009. The annexins: spatial and temporal coordination of signaling events during cellular stress. Cell. Mol. Life Sci. 66: 2623-2642.

12. Hofmann A. 2004. Annexins in the plant kingdom - perspectives and potentials. Annexins 1: $51-61$.

13. Dabitz N, Hu NJ, Yusof AM, Tranter N, et al. 2005. Structural determinants for plant annexin-membrane interactions. Biochemistry 44: 16292-16300.

14. Hu N, Yusof A, Winter A, Osman A, et al. 2008. The crystal structure of calcium-bound annexin Gh1 from Gossypium hirsutum indicates different mechanisms of membrane binding in plant and animal annexins. J. Biol. Chem. 283: 18314-18322.

15. Winter A, Yusof AM, Gao E, Yan HL, et al. 2006. Biochemical characterization of annexin B1 from Cysticercus cellulosae. FEBS J. 273: 3238-3247.

16. Römisch J, Heimburger N. 1990. Purification and characterization of six annexins from 
human placenta. Biol. Chem. Hoppe Seyler 371: 383-388.

17. Weiland M, Palm J, Griffiths W, McCaffery J, et al. 2003. Characterisation of alpha-1 giardin: an immunodominant Giardia lamblia annexin with glycosaminoglycan-binding activity. Int. J. Parasitol. 33: 1341-1351.

18. Wasmuth J, Daub J, Peregrín-Alvarez JM, Finney CAM, et al. 2009. The origins of apicomplexan sequence innovation. Genome Res 19: 1202-1213.

19. Hackett JD, Anderson D, Erdner D, Bhattacharya D.. 2004. Dinoflagellates: A remarkable evolutionary experiment. Am. J. Bot. 91: 1523-1534.

20. Dujon B, Sherman D, Fischer G, Durrens P, et al. 2004. Genome evolution in yeasts. Nature 430: 35-44.

21. Aloisio F, Filippini G, Antenucci P, Lepri E, et al. 2006. Severe weight loss in lambs infected with Giardia duodenalis assemblage B. Vet. Parasitol. 142: 154-158.

22. Geurden T, Vercruysse J, Claerebout E. 2006. Field testing of a fenbendazole treatment combined with hygienic and management measures against a natural Giardia infection in calves. Vet. Parasitol. 142: 367-371.

23. Savioli L, Smith H, Thompson A. 2006. Giardia and Cryptosporidium join the 'Neglected Diseases Initiative'. Trends Parasitol. 22: 203-208.

24. Svärd SG, Hagblom P, Palm JED. 2003. Giardia lamblia -- a model organism for eukaryotic cell differentiation. FEMS Microbiol. Lett. 218: 3-7.

25. Elmendorf HG, Dawson SC, McCaffery JM. 2003. The cytoskeleton of Giardia lamblia. Int. J. Parasitol. 33: 3-28.

26. Peattie DA, Alonso RA, Hein A, Caulfield JP. 1989. Ultrastructural localization of giardins to the edges of disk microribbons of Giardia lamblia and the nucleotide and deduced protein sequence of alpha giardin. J. Cell Biol. 109: 2323-2335.

27. Weiland M, McArthur A, Morrison H, Sogin M, et al. 2005. Annexin-like alpha giardins: a new cytoskeletal gene family in Giardia lamblia. Int. J. Parasitol. 35: 617-626. 
28. King N, Westbrook MJ, Young SL, Kuo A, et al. 2008. The genome of the choanoflagellate Monosiga brevicollis and the origin of metazoans. Nature 451: 783-788.

29. Funayama N, Nakatsukasa M, Hayashi T, Agata K. 2005. Isolation of the choanocyte in the fresh water sponge, Ephydatia fluviatilis and its lineage marker, Ef annexin. Dev. Growth Differ. 47: 243-253.

30. Srivastava M, Begovic E, Chapman J, Putnam N, et al. 2008. The Trichoplax genome and the nature of placozoans. Nature 454: 955-960.

31. Daigle SN, Creutz CE. 1999. Transcription, biochemistry and localization of nematode annexins. J. Cell Sci. 112: 1901-1913.

32. Nishioka S, Aikawa J, Ida M, Matsumoto I, et al. 2007. Ligand-binding activity and expression profile of annexins in Caenorhabditis elegans. J. Biochem. (Tokyo) 141: 47-55.

33. Creutz CE, Snyder SL, Daigle SN, Redick J. 1996. Identification, localization, and functional implications of an abundant nematode annexin. J. Cell Biol. 132: 1079-1092.

34. Creutz CE. 2003. Reflections on twenty-five years of annexin research. In: Annexins: biological importance and annexin-related pathologies. Landes Bioscience, Georgetown, TX. pp. 114.

35. Fioretti L, Warry A, Porter A, Haydock P, et al. 2001. Isolation and localisation of an annexin gene gp-nex from the potato cyst nematode Globodera pallida. Nematology 3: 45-54. 36. Ghedin E, Wang S, Spiro D, Caler E, et al. 2007. Draft genome of the filarial nematode parasite Brugia malayi. Science 317: 1756-1760.

37. Baron S. 1996. Medical microbiology. University of Texas at Galveston, Galveston, TX, USA.

38. Sinha S, Sharma B. 2009. Neurocysticercosis: a review of current status and management. $J$. Clin. Neurosci. 16: 867-876.

39. Yan H, Song Y, Fan L, Chen R, et al. 2002. Cloning and functional identification of a novel annexin subfamily in Cysticercus cellulosae. Mol. Biochem. Parasitol. 119: 1-5. 
40. Gao YJ, Yan HL, Ding FX, Lu YM, et al. 2007. Annexin B1 at the host-parasite interface of the Taenia solium cysticercus: Secreted and associated with inflammatory reaction. Acta Trop. 101: 192-199.

41. Parente L, Solito E. 2004. Annexin 1: more than an anti-phospholipase protein. Inflamm. Res. 53: 125-132.

42. Braschi S, Wilson R. 2006. Proteins exposed at the adult schistosome surface revealed by biotinylation. Mol. Cell. Proteomics 5: 347-356.

43. Mulvenna J, Moertel L, Jones MK, Nawaratna S, et al. 2010. Exposed proteins and dynamics of the Schistosoma japonicum tegument. Int. J. Parasitol. 40: 543-554.

44. King CH, Dickman K, Tisch DJ. 2005. Reassessment of the cost of chronic helmintic infection: a meta-analysis of disability-related outcomes in endemic schistosomiasis. Lancet 365: 1561-1569.

45. Steinmann P, Keiser J, Bos R, Tanner M, et al. 2006. Schistosomiasis and water resources development: systematic review, meta-analysis, and estimates of people at risk. Lancet Infect Dis 6: 411-425.

46. Jones MK, Gobert GN, Zhang L, Sunderland P, et al. 2004. The cytoskeleton and motor proteins of human schistosomes and their roles in surface maintenance and host-parasite interactions. Bioessays 26: 752-765.

47. Goldring OL, Clegg JA, Smithers SR, Terry RJ. 1976. Acquisition of human blood group antigens by Schistosoma mansoni. Clin. Exp. Immunol. 26: 181-187.

48. Mulvenna J, Sripa B, Brindley PJ, Gorman J, et al. 2010. The secreted and surface proteomes of the adult stage of the carcinogenic human liver fluke Opisthorchis viverrini. Proteomics 10: 1063-1078.

49. Berriman M, Haas BJ, LoVerde PT, Wilson RA, et al. 2009. The genome of the blood fluke Schistosoma mansoni. Nature 460: 352-358.

50. Liu F, Zhou Y, Wang Z, Lu G, et al. 2009. The Schistosoma japonicum genome reveals 
features of host-parasite interplay. Nature 460: 345-351.

51. Gobert GN, McManus DP, Nawaratna S, Moertel L, et al. 2009. Tissue specific profiling of Schistosoma japonicum by integrated laser microdissection microscopy and microarray analysis. PLoS Negl. Trop. Dis. 3: e464.

52. Choi SH, Kwon SR, Lee EH, Kim KH. 2009. Molecular cloning, functional characterization and localization of an annexin from a fish gill fluke Microcotyle sebastis (Platyhelminthes: Monogenea). Mol. Biochem. Parasitol. 163: 48-53.

53. Rand JH. 1999. Annexinopathies - A new class of diseases. New England J. Med. 340: 10351036.

54. Hajjar K, Acharya S. 2000. Annexin II and regulation of cell surface fibrinolysis. Ann. N. Y. Acad. Sci. 902: 265-271.

55. Rand JH, Arslan AA, Wu XX, Wein R, et al. 2006. Reduction of circulating annexin A5 levels and resistance to annexin A5 anticoagulant activity in women with recurrent spontaneous pregnancy losses. Am. J. Obstet. Gynecol. 194: 182-188.

56. Skrahina T, Piljic A, Schultz C. 2008. Heterogeneity and timing of translocation and membrane-mediated assembly of different annexins. Exp Cell Res 314: 1039-1047.

57. Derry MC, Sutherland MR, Restall CM, Waisman DM, et al. 2007. Annexin 2-mediated enhancement of cytomegalovirus infection opposes inhibition by annexin 1 or annexin 5. J Gen Virol 88: 19-27.

58. Gong ZJ, De Meyer S, van Pelt J, Hertogs K, et al. 1999. Transfection of a rat hepatoma cell line with a construct expressing human liver annexin V confers susceptibility to hepatitis B virus infection. Hepatology 29: 576-584.

59. Capila I, Hernaiz M, Mo Y, Mealy T, et al. 2001. Annexin V-heparin oligosaccharide complex suggests heparan sulfate-mediated assembly on cell surface. Structure 9: 57-64.

60. Ernst B, Magnani JL. 2009. From carbohydrate leads to glycomimetic drugs. Nat. Rev. Drug Discov. 8: 661-677. 
61. Calvert C, Gant S, Bowles D. 1996. Tomato annexins p34 and p35 bind to F-actin and display nucleotide phosphodiesterase activity inhibited by phospholipid binding. Plant Cell 8: 333342.

62. Hofmann A, Proust J, Dorowski A, Schantz R, et al. 2000. Annexin 24 from Capsicum annuum. X-ray structure and biochemical characterization. J. Biol. Chem. 275: 8072-8082.

63. Kaneko N, Ago H, Matsuda R, Inagaki E, et al. 1997. Crystal structure of annexin V with its ligand K-201 as a calcium channel activity inhibitor. J. Mol. Biol. 274: 16-20.

64. Hofmann A, Escherich A, Lewit-Bentley A, Benz J, et al. 1998. Interactions of benzodiazepine derivatives with annexins. J. Biol. Chem. 273: 2885-2294.

65. Kyngdon CT, Gauci CG, Gonzalez AE, Flisser A, et al. 2006. Antibody responses and epitope specificities to the Taenia solium cysticercosis vaccines TSOL18 and TSOL45-1A. Parasite Immunol. 28: 191-199.

66. Assana E, Kyngdon CT, Gauci CG, Geerts S, et al. 2010. Elimination of Taenia solium transmission to pigs in a field trial of the TSOL18 vaccine in Cameroon. Int. J. Parasitol. 40: 515519.

67. Anderson KA, Brooks AS, Morrison AL, Reid-Smith RJ, et al. 2004. Impact of Giardia vaccination on asymptomatic Giardia infections in dogs at a research facility. Can. Vet. J. 45: 924930.

68. Uehlinger FD, O'Handley RM, Greenwood SJ, Guselle NJ, et al. 2007. Efficacy of vaccination in preventing giardiasis in calves. Vet. Parasitol. 146: 182-188.

69. Nash TE. 2002. Surface antigenic variation in Giardia lamblia. Mol. Microbiol. 45: 585-590. 70. Davids BJ, Reiner DS, Birkeland SR, Preheim SP, et al. 2006. A new family of giardial cysteine-rich non-VSP protein genes and a novel cyst protein. PLoS One 1: e44.

71. Ringqvist E, Palm JED, Skarin H, Hehl AB, et al. 2008. Release of metabolic enzymes by Giardia in response to interaction with intestinal epithelial cells. Mol. Biochem. Parasitol. 159: 8591. 
72. Loukas A, Tran M, Pearson MS. 2007. Schistosome membrane proteins as vaccines. Int. J. Parasitol. 37: 257-263.

73. Cardoso FC, Pacfico RNA, Mortara RA, Oliveira SC. 2006. Human antibody responses of patients living in endemic areas for schistosomiasis to the tegumental protein Sm29 identified through genomic studies. Clin. Exp. Immunol. 144: 382-391.

74. Cardoso FC, Pinho JMR, Azevedo V, Oliveira SC. 2006. Identification of a new Schistosoma mansoni membrane-bound protein through bioinformatic analysis. Genet. Mol. Res. 5: 609-618.

75. Tran M, Pearson M, Bethony J, Smyth D, et al. 2006. Tetraspanins on the surface of Schistosoma mansoni are protective antigens against schistosomiasis. Nature Med. 12: 835-840. 76. Loukas A, Bethony J, Brooker S, Hotez P. 2006. Hookworm vaccines: past, present, and future. Lancet Infect Dis 6: 733-741.

77. Nolan MJ, Hofmann A, Jex AR, Gasser RB. 2010. A theoretical study to establish the relationship between the three-dimensional structure of triose-phosphate isomerase of Giardia duodenalis and point mutations in the respective gene. Mol. Cell. Probes in press: .

78. Adam RD. 2001. Biology of Giardia lamblia. Clin. Microbiol. Rev. 14: 447-475.

79. Nolan MJ, Jex AR, Pangasa A, Young ND, et al. 2010. Analysis of nucleotide variation within the triose-phosphate isomerase gene of Giardia duodenalis from sheep and its zoonotic implications. Electrophoresis 31: 287-298.

80. de Lourdes Mottier M, Prichard RK. 2008. Genetic analysis of a relationship between macrocyclic lactone and benzimidazole anthelmintic selection on Haemonchus contortus. Pharmacogenet. Genomics 18: 129-140.

81. von Samson-Himmelstjerna G, Walsh TK, Donnanm A A, Carrière S, et al. 2009. Molecular detection of benzimidazole resistance in Haemonchus contortus using real-time PCR and pyrosequencing. Parasitology 136: 349-358.

82. Kopp SR, Coleman GT, Traub RJ, McCarthy JS, et al. 2009. Acetylcholine receptor 
subunit genes from Ancylostoma caninum: altered transcription patterns associated with pyrantel resistance. Int. J. Parasitol. 39: 435-441.

83. Kasinathan RS, Goronga T, Messerli SM, Webb TR, et al. 2010. Modulation of a Schistosoma mansoni multidrug transporter by the antischistosomal drug praziquantel. FASEB J. 24: 128-135.

84. Messerli SM, Kasinathan RS, Morgan W, Spranger S, et al. 2009. Schistosoma mansoni Pglycoprotein levels increase in response to praziquantel exposure and correlate with reduced praziquantel susceptibility. Mol. Biochem. Parasitol. 167: 54-59.

85. Cai P, Bu L, Wang J, Wang Z, et al. 2008. Molecular characterization of Schistosoma japonicum tegument protein tetraspanin-2: sequence variation and possible implications for immune evasion. Biochem. Biophys. Res. Commun. 372: 197-202.

86. Schistosoma japonicum Genome Sequencing and Functional Analysis Consortium. 2009. The Schistosoma japonicum genome reveals features of host-parasite interplay. Nature 460: 345351.

87. Sali A, Blundell T. 1993. Comparative protein modelling by satisfaction of spatial restraints. J. Mol. Biol. 234: 779-815.

88. Laskowski R, MacArthur M, Moss D, Thornton J. 1993. PROCHECK: A program to check the stereochemical quality of protein structures. J. Appl. Cryst. 26: 283-291.

89. Weng X, Luecke H, Song IS, Kang DS, et al. 1993. Crystal structure of human annexin I at 2.5 A resolution. Protein Sci. 2: 448-458.

90. Sopkova J, Renouard M, Lewit-Bentley A. 1993. The crystal structure of a new highcalcium form of annexin V. J. Mol. Biol 234: 816-825.

91. Pathuri P, Nguyen ET, Svard SG, Luecke H. 2007. Apo and Calcium-bound Crystal Structures of Alpha-11 Giardin, an Unusual Annexin from Giardia lamblia. J. Mol. Biol. 368: 493508.

92. Pathuri P, Nguyen ET, Ozorowski G, Svärd SG, et al. 2009. Apo and calcium-bound crystal 
structures of cytoskeletal protein alpha-14 giardin (annexin E1) from the intestinal protozoan parasite Giardia lamblia. J. Mol. Biol. 385: 1098-1112.

93. Kumar S, Tamura K, Nei M. 2004. MEGA3: Integrated software for Molecular Evolutionary Genetics Analysis and sequence alignment. Brief Bioinform. 5: 150-163.

94. Bryson K, McGuffin LJ, Marsden RL, Ward JJ, et al. 2005. Protein structure prediction servers at University College London. Nucl. Acids Res. 33: W36-W38. 
Table 1: Accession numbers of parasite annexins

\begin{tabular}{|c|c|c|c|c|c|}
\hline $\begin{array}{l}\text { Annexin } \\
\text { nomenclature } \\
\text { in this manuscript }\end{array}$ & Species & $\begin{array}{l}\text { No of } \\
\text { amino } \\
\text { acids }\end{array}$ & GenBank $^{1}$ & SchistoDB $^{2}$ or GiardiaDB ${ }^{3}$ & Other accession numbers \\
\hline ANXB1(Ts) & T. solium & 346 & AAF64166 & & \\
\hline ANXB2(Ts) & T. solium & 354 & AAY17503 & & \\
\hline ANXB3(Ts) & T. solium & 310 & AAY27744 & & \\
\hline ANXB13(Bm) & B. mori & 486 & BAB78533 & & NP_001036881 \\
\hline ANX(Sm)1 & S. mansoni & 365 & AAC79802 & Smp_074150 & \\
\hline ANX(Sm)2 & S. mansoni & 352 & CAZ35184 & Smp_164100 & XP_002578946 \\
\hline ANX(Sm)3 & S. mansoni & 340 & ACO90180 & Smp_077720 & XP_002578945 \\
\hline $\mathrm{ANX}(\mathrm{Sm}) 4$ & S. mansoni & 331 & CAZ32112 & Smp_045560 & XP_002575877 \\
\hline ANX(Sm)5 & S. mansoni & 287 & CAZ34823 & Smp_074140 & XP_002578585 \\
\hline ANX(Sm)6 & S. mansoni & 344 & CAZ34822 & Smp_162160 & XP_002578584 \\
\hline ANX(Sm)7 & S. mansoni & 398 & CAZ33727 & Smp_155580.1, Smp_155580.2 & XP_002577489 \\
\hline $\mathrm{ANX}(\mathrm{Sm}) 8$ & S. mansoni & 349 & CAZ35198 & Smp_077880.2 & XP_002578960 \\
\hline ANX(Sm)9 & S. mansoni & 545 & CAZ32106 & Smp_045500 & XP_002575871 \\
\hline ANX(Sm)10 & S. mansoni & 849 & CAZ32110 & Smp_146680 & XP_002575875 \\
\hline ANX(Sm)11 & S. mansoni & 508 & CAZ32105 & Smp_045490 & XP_002575870 \\
\hline $\mathrm{ANX}(\mathrm{Sm}) 12$ & S. mansoni & 487 & CAZ32111 & Smp_045550 & XP_002575876 \\
\hline CAZ32113 & S. mansoni & 706 & CAZ32113 & Smp_146690 & XP_002577489 \\
\hline $\mathrm{ANX}(\mathrm{Sj}) 1$ & S. japonicum & 359 & AAW25344 & & AY813612 \\
\hline $\mathrm{ANX}(\mathrm{Sj}) 2$ & S. japonicum & 346 & AAP06415 & & AJ306451 \\
\hline ANX(Sj)3 & S. japonicum & 340 & AAW24721 & & AY812989 \\
\hline ANX(Sj)4 & S. japonicum & 330 & AAP06504 & & AY223469 \\
\hline ANX(Sj)5 & S. japonicum & 354 & AAW26786 & & AY815054 \\
\hline ANX(Sj)7 & S. japonicum & 374 & AAW26872 & & AY815140 \\
\hline ANX(Ef) & E. fluviatilis & 321 & BAD99420 & & \\
\hline ANX(Ms) & M. sebastis & 353 & ACD93001 & & EU719209 \\
\hline ANX(Ta) 1 & T. adhaerens & 318 & EDV27661 & & XP_002109495 \\
\hline $\mathrm{ANX}(\mathrm{Ta}) 2$ & T. adhaerens & 292 & EDV20685 & & XP_002116885 \\
\hline ANX(Ta)3 & T. adhaerens & 323 & EDV23675 & & XP_002113201 \\
\hline ANX(Hg) & H. glycines & 341 & AAN32888 & & \\
\hline ANX(Bm)1 & B. malayi & 255 & EDP35210 & & XP_001895943 \\
\hline ANX(Bm)2 & B. malayi & 381 & EDP37127 & & XP_001894032 \\
\hline nex-1 & C. elegans & 322 & AAB52702 & & WBGene00003588 \\
\hline nex-2 & C. elegans & 455 & NP_001022756 & & WBGene00003589 \\
\hline nex-3 & C. elegans & 317 & NP_497903 & & WBGene00003590 \\
\hline nex-4 & C. elegans & 351 & NP_504300.1 & & WBGene00003591 \\
\hline Alpha-1 giardin & G. lamblia & 295 & AAX07975 & GL50803_11654 & AY781324 \\
\hline Alpha-2 giardin & G. lamblia & 296 & AAA29150 & GL50803_7796 & AY781317 \\
\hline Alpha-3 giardin & G. lamblia & 296 & AAL30503 & GL50803_11683 & AY781325 \\
\hline
\end{tabular}




\begin{tabular}{|l|l|l|l|l|l|} 
Alpha-4 giardin & G. lamblia & 296 & AAX07970 & GL50803_7799 & AY781319 \\
\hline Alpha-5 giardin & G. lamblia & 302 & AAX07969 & GL50803_7797 & AY781318 \\
\hline Alpha-6 giardin & G. lamblia & 297 & AAX07978 & GL50803_14551 & AY781327 \\
\hline Alpha-7.1 giardin & G. lamblia & 388 & AAX07982 & GL50803_103373 & AY781331 \\
\hline Alpha-7.2 giardin & G. lamblia & 388 & AAX07984 & GL50803_114119 & AY781333 \\
\hline Alpha-7.3 giardin & G. lamblia & 295 & AAX07985 & GL50803_114787 & AY781334 \\
\hline Alpha-8 giardin & G. lamblia & 311 & AAX07974 & GL50803_11649 & AY781323 \\
\hline Alpha-9 giardin & G. lamblia & 301 & AAX07983 & GL50803_103437 & AY781332 \\
\hline Alpha-10 giardin & G. lamblia & 328 & AAX07967 & GL50803_5649 & AY781316 \\
\hline Alpha-11 giardin & G. lamblia & 307 & AAX07981 & GL50803_17153 & AY781330 \\
\hline Alpha-12 giardin & G. lamblia & 323 & AAX07973 & GL50803_10073 & AY781322 \\
\hline Alpha-13 giardin & G. lamblia & 345 & AAX07965 & GL50803_1076 & AY781314 \\
\hline Alpha-14 giardin & G. lamblia & 337 & AAX07979 & GL50803_15097 & AY781328 \\
\hline Alpha-15 giardin & G. lamblia & 303 & AAX07977 & GL50803_13996 & AY781326 \\
\hline Alpha-16 giardin & G. lamblia & 365 & AAX07971 & GL50803_10036 & AY781320 \\
\hline Alpha-17 giardin & G. lamblia & 310 & AAX07980 & GL50803_15101 & AY781329 \\
\hline Alpha-18 giardin & G. lamblia & 286 & AAX07972 & GL50803_10038 & AY781321 \\
\hline Alpha-19 giardin & G. lamblia & 438 & AAX07966 & GL50803_4026 & AY781315 \\
\hline
\end{tabular}

${ }^{1}$ Protein accession number at http://www.ncbi.nlm.nih.gov/Genbank

${ }^{2}$ Gene Name at http://www.genedb.org/

${ }^{3}$ GeneID at http://giardiadb.org/giardiadb/ 


\section{Figure Legends}

\section{Figure 1}

Three-dimensional model of $T$. solium annexin B1 obtained by comparative modelling. Figure prepared with PyMOL (DeLano W. 2002. The PyMOL Molecular Graphics System. http://www.pymol.org).

The crystal structure of human annexin A8 (PDB accession code 1w3w) was chosen as a template to model T. solium annexin B1 based on its amino acid sequence identity (40\%) which was found to be the highest of all annexins with known three-dimensional structure. A structure-based amino acid sequence alignment of the two proteins was obtained as described above, and subjected to comparative modelling calculations with MODELLER.[87] Twenty independent models were computed, restraining the predicted $\alpha$-helical segment in the linker region to $\alpha$-helical geometry. The model with the lowest energy was selected, and the overall geometry was scrutinized with PROCHECK.[88] The N-terminal first 23 amino acid residues are not present in the model due to the absence of the N-terminal domain in the template.

Annexin proteins are characterised by a fourfold repeat (I-IV) of a 70 amino acid sequence folding into a four-helix barrel (helices A, B, D and E) that is capped by a fifth $\alpha$-helix (C) perpendicular to the barrel (indicated in repeat I).[7] At the top of the barrel, the connecting loops between helices $\mathrm{A} / \mathrm{B}$ and $\mathrm{D} / \mathrm{E}$ harbour binding sites for calcium ions that mediate the binding of the protein molecules to acidic phospholipid membranes. To distinguish these binding sites from the ones found in EF-hand proteins (type I), they have been termed type II (in A/B loops) and type III sites (in D/E loops).[89] At high concentrations of metal ions, a second metal ion can be bound in the A/B loop, in a site called AB'.[90] Recent structural investigation of alpha-11 and alpha-14 giardin revealed that the annexin fold also allows for a fourth type of calcium coordination in the D/E loop, in a site termed type IIIb.[91,92] The predicted $\alpha$-helical element in the linker region between repeats II and III (shown in red) is a unique feature shared by many parasite annexins. 


\section{Figure 2}

Minimum evolution phylogenetic tree of selected invertebrate annexins. For accession numbers see Table 1.

Multiple sequences were aligned using the Clustal W1.8 algorithm on the EBI server (http://www.ebi.ac.uk/Tools/clustalw2/index.html). Phylogenetic analysis was performed using MEGAv4.0 software for Molecular Evolutionary Genetics Analysis[93] to compare annexin sequences from selected lower metazoans. The sequences were identified from public databases by BLAST searches. A minimum evolution phylogenetic tree was constructed using the JTT substitution model, and uniform rates among sites were assumed. The dataset was bootstrapped 1000 times, with the resulting percentage values shown on branches of the tree. The tree is midpoint-rooted.

\section{Figure 3}

Structure-based amino acid sequence alignment of the linker region between repeats II and III of alpha giardins. The predicted $\alpha$-helical secondary structure elements are indicated in green. For accession numbers see Table 1.

Genbank (http://www.ncbi.nlm.nih.gov/Genbank), the schistosome (http://www.schistodb.org) and Giardia (http://giardiadb.org) databases were mined for annexins from individual organisms. Secondary structure elements of proteins were predicted using PSIPRED,[94] and structure-based sequence alignments were generated manually. The nomenclature used for parasite annexins in this manuscript is for temporary reference only.

\section{Figure 4}

Structure-based amino acid sequence alignment of the linker region between repeats II and III of selected invertebrate annexins. Predicted[94] $\alpha$-helical secondary structure elements are indicated in 
green. For accession numbers see Table 1. Note that sequences represent putative predictions derived from automated genomic analyses. 
Figure 1

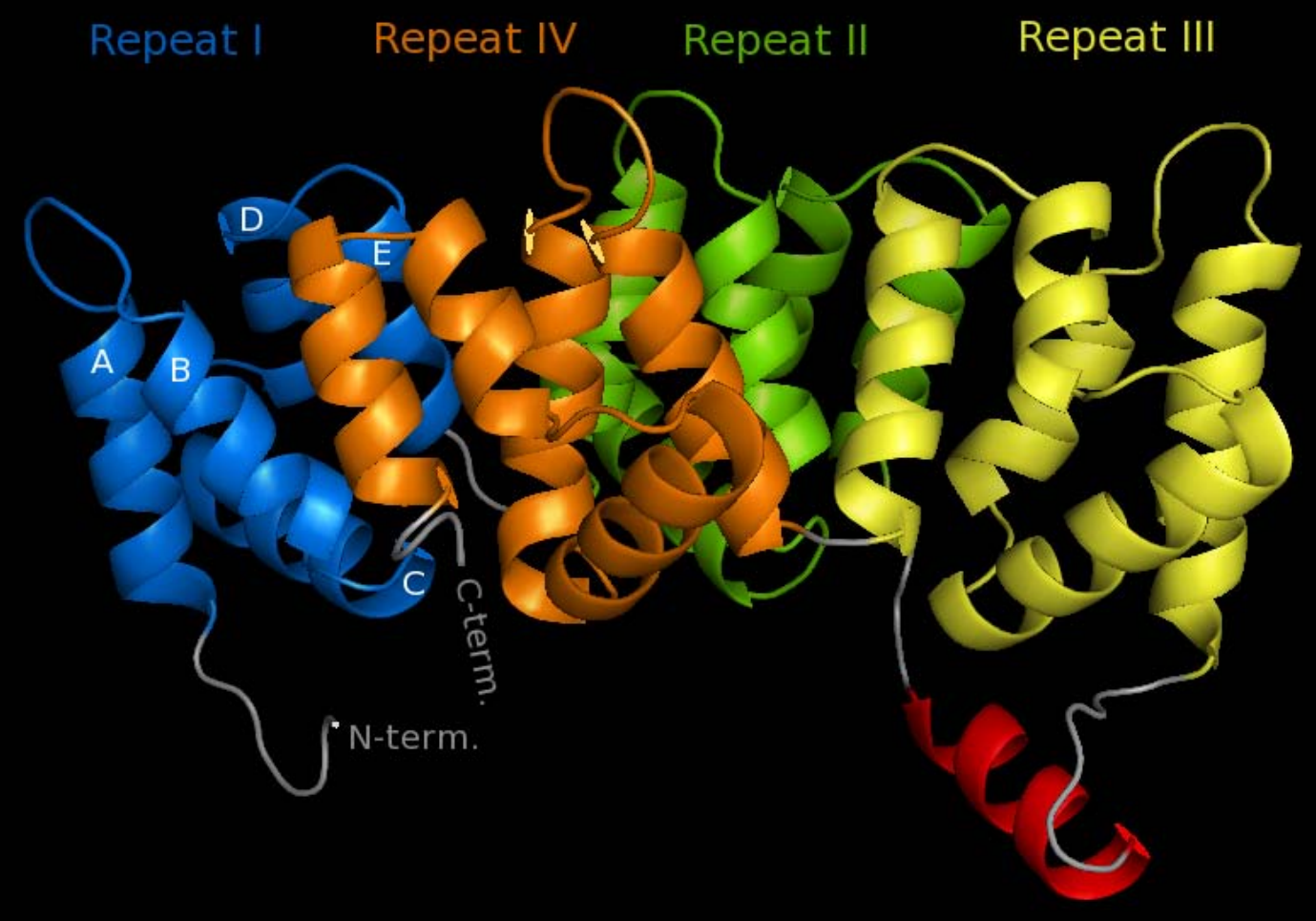


Figure 2

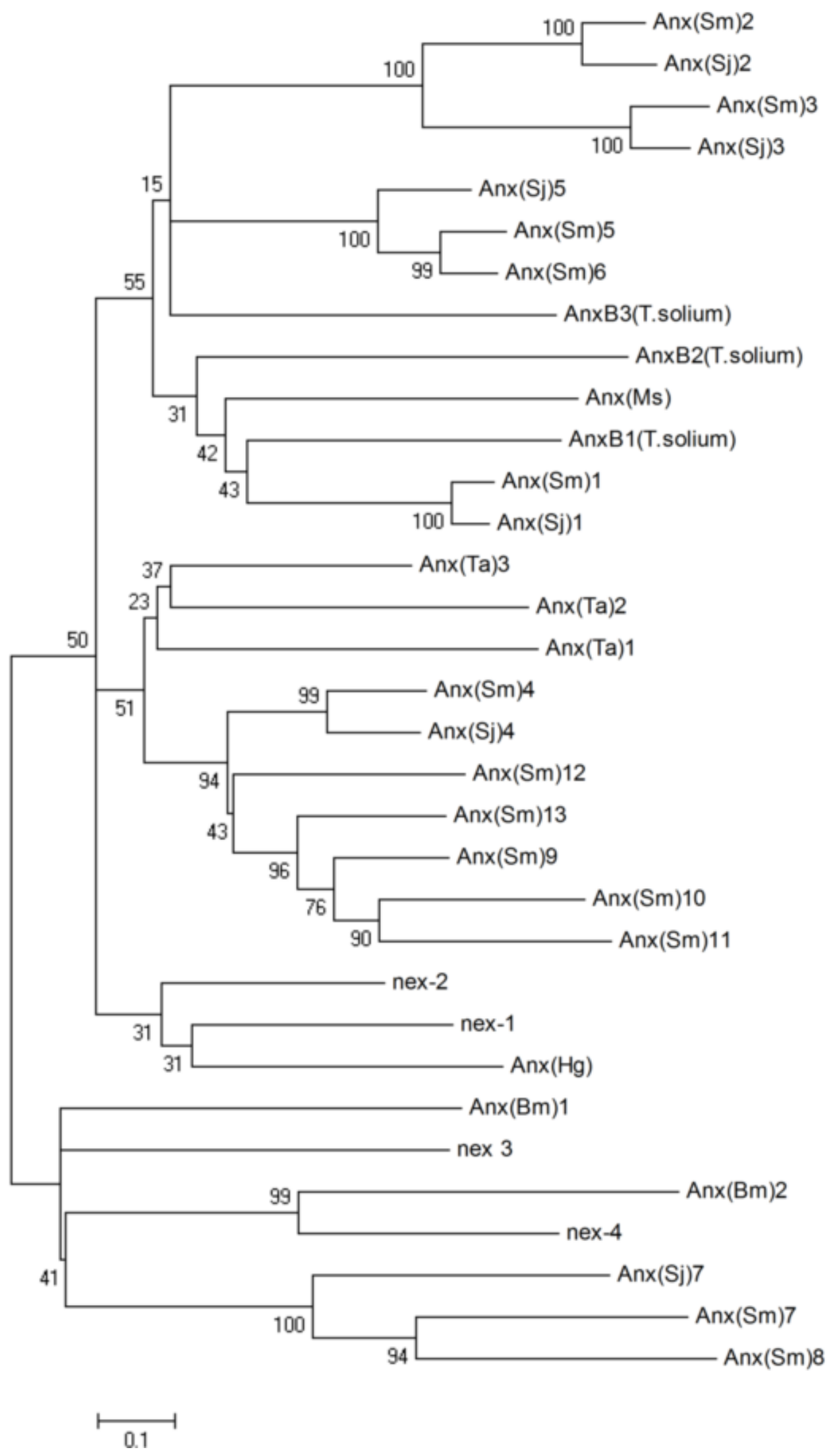


Figure 3

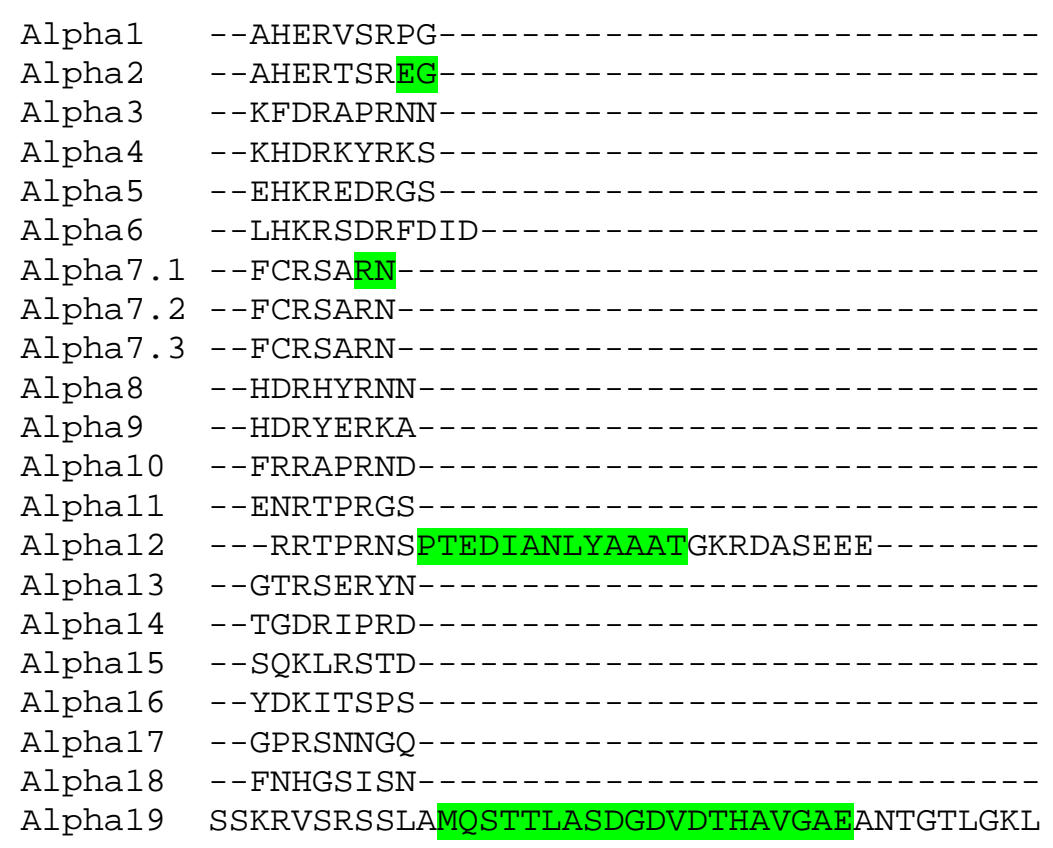




\section{Figure 4}

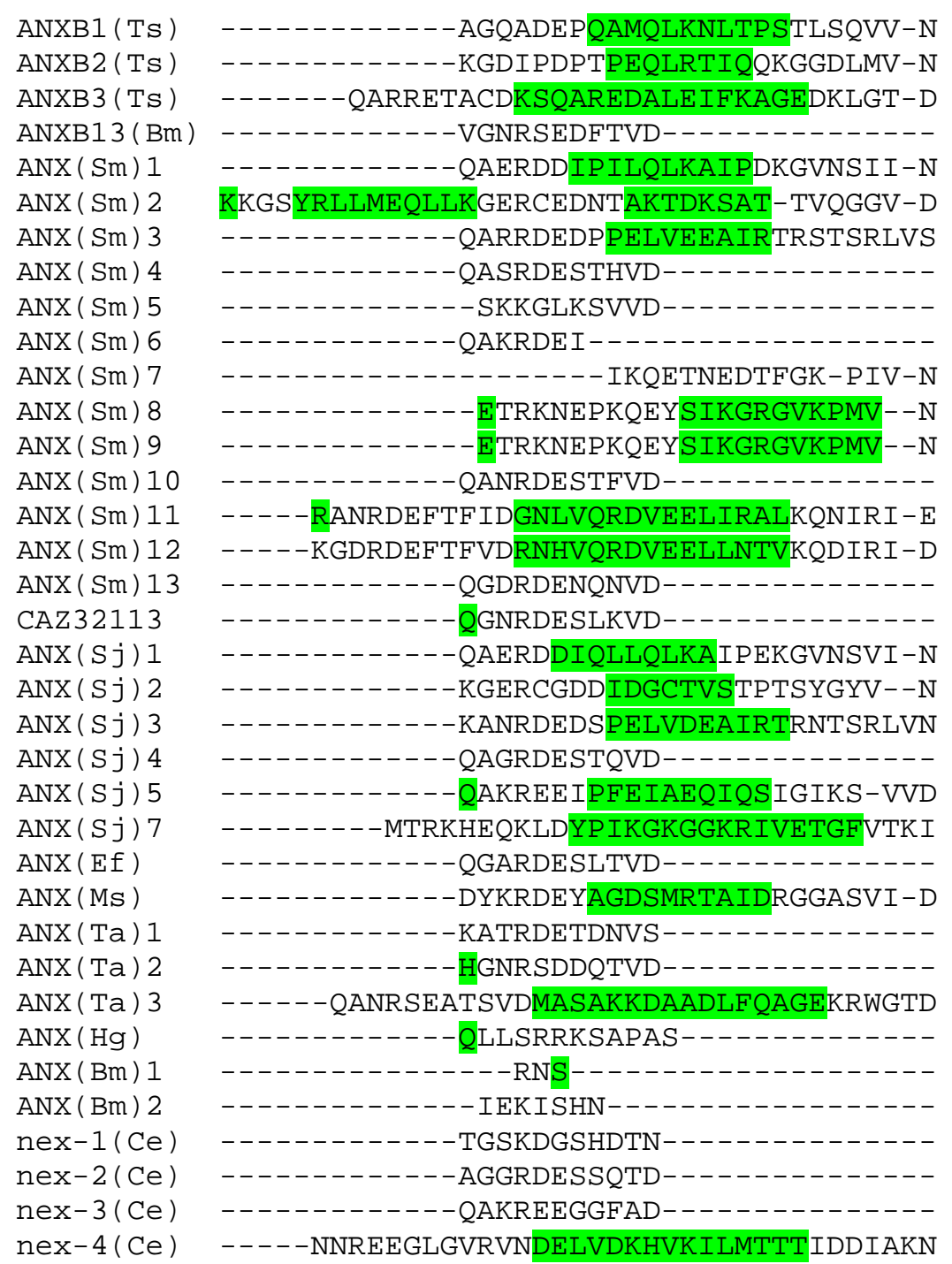

